\title{
Diseño de un Sendero Ecológico como Estrategia Pedagógica para Promover el Conocimiento del Ecosistema de Manglar en la Escuela Naval de Cadetes "Almirante Padilla"
}

\section{Design of an Ecological Route as a Pedagogical Strategy to Promote the Knowledge of the Mangrove Ecosystem in the Naval School of Cadets "Almirante Padilla"}

Cristhian Mendoza-Pérez*

Escuela Naval de Cadetes "Almirante Padilla" - Colombia

ORCID iD: https://orcid.org/0000-0002-7885-815X

poseidon@enap.edu.co

\section{Marena Vitola-Quintero}

Escuela Naval de Cadetes "Almirante Padilla" - Colombia ORCID iD: https://orcid.org/0000-0001-5219-3615

marena.vitola@enap.edu.co

Valeria Rojas-Sáenz

Escuela Naval de Cadetes "Almirante Padilla" - Colombia valeria.rojas@armada.mil.co

\section{Danna Valencia-Rosas}

Escuela Naval de Cadetes "Almirante Padilla" - Colombia danna.valencia@armada.mil.co

* Autor a quien debe ser dirigida la correspondencia

\section{Resumen}

El presente trabajo es el resultado de un levantamiento de información en campo orientado a identificar las características ecológicas del ecosistema de manglar presente en inmediaciones de la Escuela Naval de Cadetes "Almirante Padilla", tales como especies de mangle presentes en el área de estudio y su fauna asociada. Información relevante para diseñar un sendero ecológico que logre mostrar en toda su dimensión la relevancia del bosque de manglar que va desde la gran biodiversidad que alberga hasta la protección que brinda a las costas contra desastres naturales y la erosión. El tipo de investigación fue definida como exploratoria (Ávila 2006), ya que es la primera propuesta de esta naturaleza en la ENAP, con un enfoque cualitativo (Fernández, 2000) toda vez que la información levantada tiene dicho origen; cabe anotar que para el diseño del sendero se desarrollaron los siguientes pasos: 1) Reconocimiento del área de estudio; 2) Identificación de las estaciones de observación; 3) Elaboración del esquema de sendero ecológico. Teniendo en cuenta lo anterior el objetivo de la investigación se centra en la construcción de una herramienta pedagógica (sendero ecológico) orientada a dar a conocer "in situ" todos los beneficios que brinda el ecosistema de manglar al medio ambiente y la sociedad en general así como sus características, propendiendo por su conservación y uso sostenible en el tiempo.

\section{Palabras clave:}

Manglar; sendero ecológico; beneficios; herramienta pedagógica; ecosistema.

\begin{abstract}
The present work is the result of an information gathering in the field oriented to identify the ecological characteristics of the mangrove ecosystem present near the Navy School "Almirante Padilla", such as mangrove species present in the study area and its associated fauna. Relevant information to design an ecological path that manages to show in all its dimensions the relevance of the mangrove forest that goes from the great biodiversity it houses to the protection it provides to the coasts against natural disasters and erosion. The type of research was defined as exploratory (Ávila 2006), since it is the first proposal of this nature in the ENAP, with a qualitative approach (Fernández, 2000) since the information collected
\end{abstract}


has that origin; It should be noted that the following steps were developed for the design of the path: 1) Recognition of the study area; 2) Identification of observation stations; 3) Development of the ecological path scheme. Taking into account the above, the objective of the research is focused on the construction of a pedagogical tool (ecological path) aimed at making known "in situ" all the benefits that the ecosystem of mangrove brings to the environment and society in general, provided for its conservation and sustainable use over time.

Keywords:

Mangrove; ecological path; benefits; educational tool; ecosystem.

\section{Introducción}

Los bosques de manglar son uno de los ecosistemas más productivos en el planeta, y de ese modo también son los bosques más amenazados por la deforestación, incluso en Colombia el $50 \%$ de los boques de mangles han enfrentado esta misma situación (Ministerio de Medio Ambiente, 2002). Así mismo, el mangle es de vital importancia para la biodiversidad. Proporcionan ricos recursos pesqueros y protegen a las comunidades contra las tormentas y la erosión. Sin embargo, por su localización en la zona intermareal, es un ecosistema afectado frente al cambio climático global, afectando su función como componente fundamental en los estuarios tropicales y la interfase entre la tierra y el mar; por lo tanto, son muy sensitivos a los cambios en el nivel del mar. Los suelos en los que se desarrollan los mangles son generalmente ácidos por la alta descomposición de materia orgánica, si los suelos de estos mangles son removidos o dragados, los depósitos de azufre son liberados, lo que podrá causar condiciones de acidez extrema en el medio (Fundación Universitaria Tecnológico Comfenalco, 2010)

En ese contexto, el incremento en las temperaturas, asociado al calentamiento global, ha provocado una disminución en el crecimiento de los manglares. La intensidad de los rayos solares, advirtió Rafael Riosmena, investigador de la UABCS, ha empezado a afectar los periodos de floración de los humedales, con lo que se está evitando el nacimiento de nuevos miembros a las comunidades. Este problema, también afecta a algunas especies de la flora marina, las cuales se están viendo afectados, puesto que su permanencia depende de la supervivencia de los árboles adultos (Riosmena,
2015). Sumado a lo descrito se encuentra el efecto antrópico al cual se encuentran sometidos este tipo de ecosistemas, el cual ocasiona daños irreparables en la dinámica de mismos, en su mayoría de las veces por el desconocimiento de las bondades que el bosque de manglar ofrece al medio ambiente y a las comunidades en general.

Teniendo en cuenta lo anterior, la presente investigación es motivada por la necesidad de acercamiento entre la comunidad y el manglar, para de esta manera conocer de primera mano los tipos de mangle presentes en la Isla de Manzanillo, así como la fauna asociada a estas plantas, lo que en conjunto contribuye a crear conciencia a través de una herramienta pedagógica como lo es el sendero ecológico, que permite "in situ" conocer los beneficios del ecosistema provocando la conservación y uso sostenible en el tiempo de tan importante interés marítimo de la nación.

\section{Metodología}

La presente propuesta se enmarcó en el tipo de investigación exploratoria que, según Ávila (2006), trata de conocer el tema que se abordó, para que se familiaricen con algo que hasta el momento desconocían. Los resultados de este tipo de investigación dan un panorama o conocimiento superficial del tema, pero es el primer paso para cualquier tipo de investigación posterior que se llevó a cabo. Cabe anotar que el enfoque de la investigación es de carácter cualitativo. Centrado en la identificación de la naturaleza profunda de las realidades, su sistema de relaciones y su estructura dinámica (Fernández, 2002). 
Diseño de un Sendero Ecológico como Estrategia Pedagógica para Promover el Conocimiento del Ecosistema de Manglar en la Escuela Naval de Cadetes "Almirante Padilla"

Para alcanzar los objetivos de investigación se desarrolló el siguiente paso a paso:

Reconocimiento del área de estudio: $\mathrm{Se}$ realizó una revisión de literatura y análisis documental (Quintana y Montgomery, 2006) en bases de datos reconocidas y de acceso público como Scielo, Dialnet, Google académico, Google libros y Sistema de Gestión del Conocimiento, así como los documentos encontrados en el derrotero de la Escuela Naval (Mendoza y Vitola, 2014), sobre estudios relacionados llevados a cabo en el Caribe, Cartagena e Isla de Manzanillo, acerca de las especies de fauna y flora más relevantes del ecosistema de manglar, así como datos de clima de la zona de estudio como pluviosidad, intervención antrópica y cambios en el paisaje a lo largo del tiempo, con el objetivo de obtener de manera integral la información más relevante del ecosistema y así conocer los tópicos temáticos se pueden ofrecer en cada estación de observación durante el recorrido del sendero.

Posteriormente, se llevaron a cabo dos salidas de campo, en las cuales se pretendió conocer de primera mano las características del área que ocupa el ecosistema actualmente en términos de fácil acceso a la zona del sendero, seguridad, verificar que el área del sendero no sea inundable y que el recorrido que se vaya a diseñar incluya buena parte del ecosistema de manglar, con el objetivo de garantizar que el sendero aporte la mayor cantidad de información ecológica al personal que haga uso del mismo. Se resalta que para la observación en campo de los elementos bióticos y abióticos que hacen parte del manglar se llevará a cabo de acuerdo a lo planteado por Fuentes (2015) quien supone que las salidas de campo constituyen una oportunidad importante para obtener información "in situ" de las entidades de estudio, de su hábitat, ecología, poblaciones, interacciones, amenazas, usos, etc. Información que fue recopilada en el cuaderno de campo.

Identificación de las estaciones de observación: Con base en la información levantada "in situ" se procedió a identificar cada una de las estaciones de observación que harán parte del sendero ecológico a diseñar, teniendo en cuenta fácil acceso, condiciones favor- ables para el recorrido, la facilidad para la observación de especies vegetales y animales propias del ecosistema, describiendo en cada estación la actividad reflexiva que se llevará a cabo en términos de reconocimiento de flora y fauna endémica del ecosistema, de acuerdo a lo planteado por Lechner (2004).

Así mismo se delimitó el área del sendero, identificando los lugares potenciales para la implementación analizando fortalezas y debilidades; evaluando los factores ambientales y valorando por donde se puede desarrollar, no obstante, se identificaron los impactos que este generaría ya sea positivo o negativo bien sea en el paisaje, en la flora y/o en la fauna; por ende, es importante tener en cuenta las zonas de riesgo del sendero como deslizamiento del terreno, humedales, hundimientos etc. No obstante, una vez definidos los puntos anteriores se analizará si va a ser un sendero lineal, tipo circuito o multicircuito (Larbanois, 2004).

Elaboración del esquema que ilustre el sendero ecológico: Se establecieron puntos en el sendero escogidos por su mayor biodiversidad siendo lugares accesibles y oportunos. Cada punto del sendero se escogió con el fin de resaltar la formación y dinámica del ecosistema, el efecto antrópico en la zona, explicando los cuidados que conlleva cada tipo de manglar presente en la Escuela Naval, así como la posible fauna asociada a cada uno.

Posteriormente el sendero se diseñó con el programa de AutoCAD, que es un programa de dibujo por computadora para generar esquemas en 2 y 3 dimensiones, pudiendo crear dibujos o planos genéricos, documentar proyectos de ingeniería, arquitectura, mapas o sistemas de información geográfica por mencionar algunas industrias y aplicaciones (Autocad, 1982).

Con el fin de dejar el sendero estructurado y con las especificaciones que se requiere, identificando cada estación de observación a lo largo del recorrido, por medio de coordenadas; como los puntos de salida y llegada.

Finalmente, se utilizó el software Adobe Photoshop para superponer el esquema diseñado en una imagen satelital del área de estudio 
obtenida de Google Earth (2019), para de esta manera tener una idea clara del área que comprenderá el sendero en inmediaciones de la ENAP.

\section{Resultados}

A continuación, se muestra la información levantada en campo reflejada en registro fotográfico tomado en la zona de estudio, la cual constituyó el insumo para el diseño del sendero ecológico constituido por cinco estaciones de observación siendo del tipo lineal según con punto de inicio y final en diferentes zonas según lo descrito por Lorbanois (2004):
La primera estación del sendero (coordenadas $10^{\circ} 23^{\prime} 17^{\prime}$ N $75^{\circ} 31^{\prime} 38^{\prime \prime} \mathrm{W}$ ) (Figura 2) se eligió por su fácil acceso, ya que presenta amplio espacio para impartir las tareas e instrucciones que se desarrollaran en el recorrido (Figura 2), en esta estación se visualizaron guaridas cangrejos mangleros (Minuca rapax), y el mangle zaragoza (Conocarpus erectus) (Figura 1) que generalmente no mide más de $10 \mathrm{~m}$ de altura. No se considera un mangle verdadero, sino más bien una especie que crece en partes elevadas con sustratos arenosos y menos salados. Se caracterizan también por producir un pequeño fruto que parece una piña (Mejía et al., 2014).

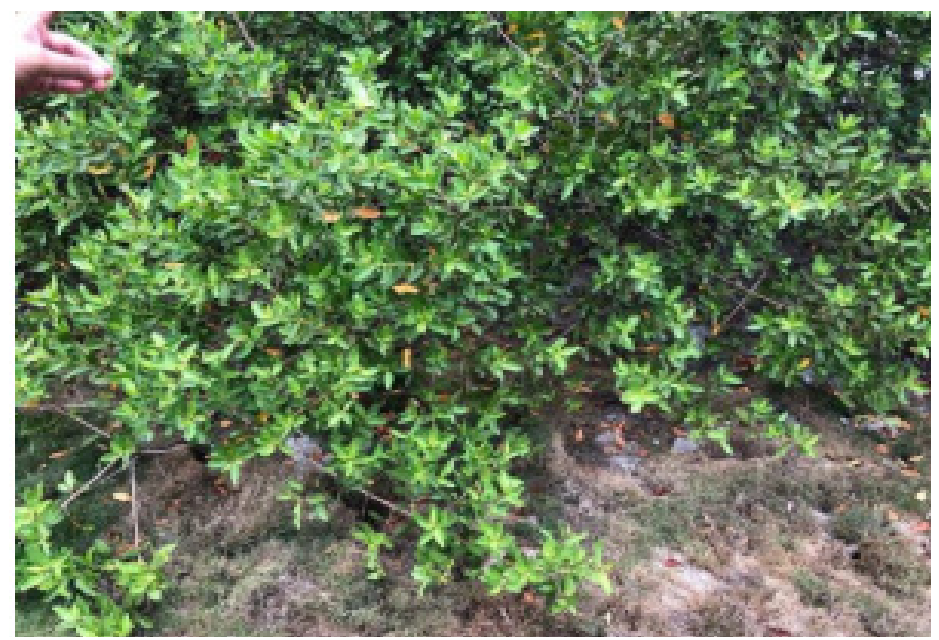

Figura 1. Mangle zaragoza

Fuente: Elaboración propia

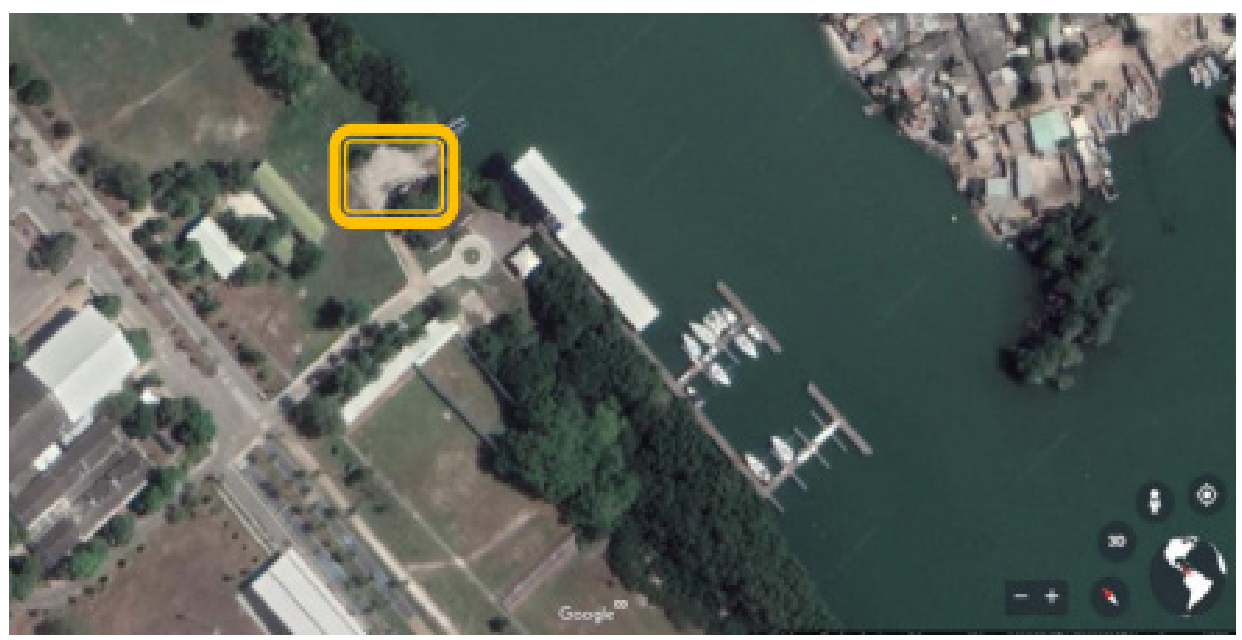

Figura 2. Primera estación

Fuente: Elaboración propia con base en Google Earth (2019) 
Diseño de un Sendero Ecológico como Estrategia Pedagógica para Promover el Conocimiento del Ecosistema de Manglar en la Escuela Naval de Cadetes "Almirante Padilla"

En La segunda estación $\left(10^{\circ} 23^{\prime} 15^{\prime \prime} \mathrm{N}\right.$ dos propágulos. Los árboles pueden llegar a 75'31'38”W) (Figura 4) se encontró presencia medir $45 \mathrm{~m}$ de altura en las desembocaduras de de mangle rojo (Rhizophora mangle), como se los ríos, tienen una corteza de color gris claro visualiza en la Figura 3 se reconoce fácilmente con manchas oscuras. Su madera no tiene anilporque tiene raíces en forma de zanco y se re- los de crecimiento y es de color rojizo (Mejía produce con unas estructuras alargadas llama- et al., 2014).

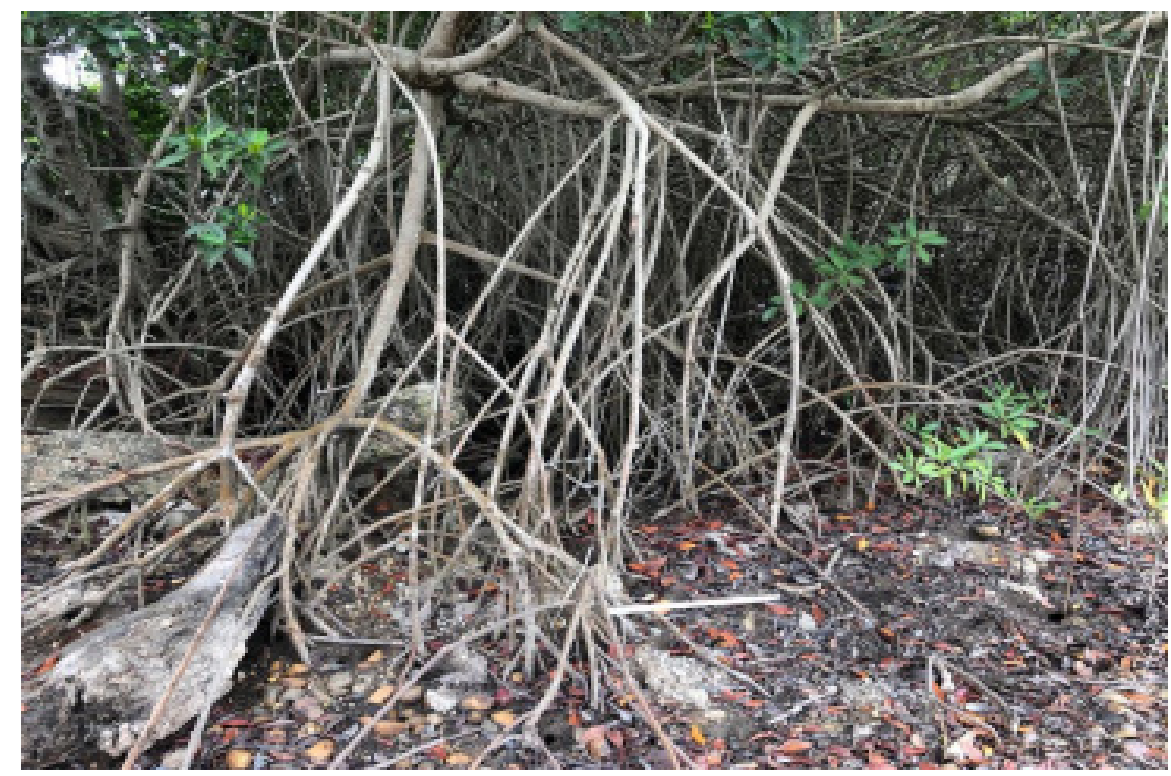

Figura 3. Mangle rojo

Fuente: Elaboración propia

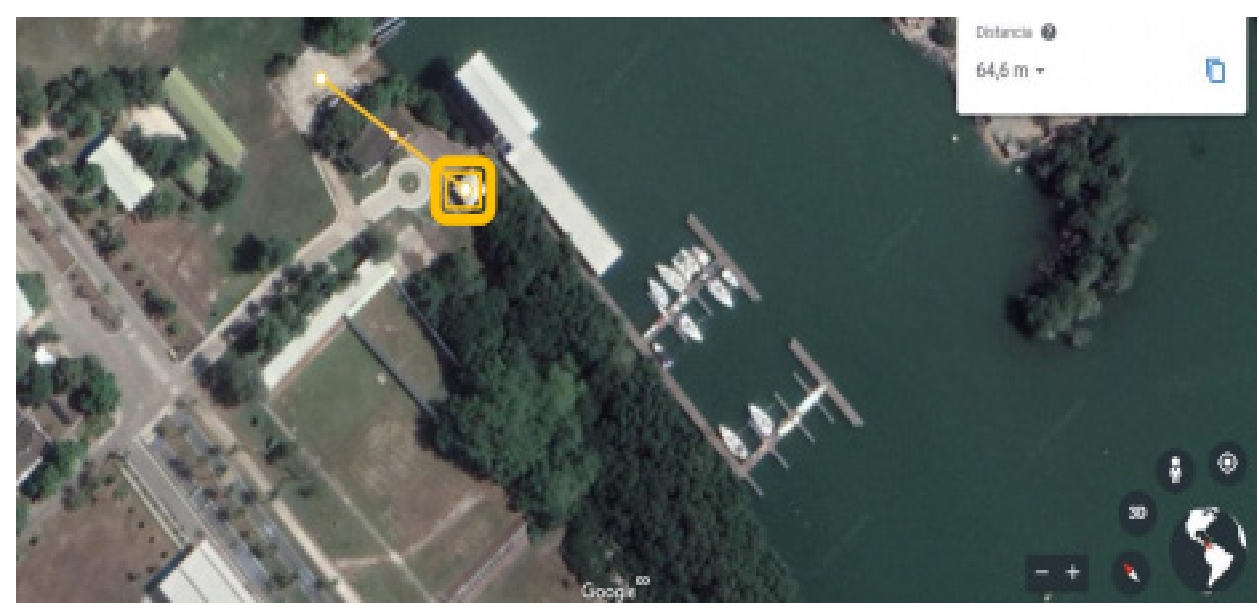

Figura 4. Segunda estación

Fuente: Elaboración propia con base en Google Earth (2019)

En la tercera estación (Coordenadas $10^{\circ}$ 23 ' $11^{\prime \prime} \mathrm{N} 75^{\circ} 31^{\prime} 38^{\prime \prime} \mathrm{W}$ ) se encuentra en medio del bosque de manglar (Figura 6) donde se pueden encontrar mapaches (Procyon cancrivorus), iguanas (Iguana iguana) y mangle blanco (Laguncularia racemosa) el cual tiene unas glándulas en la base de las hojas que le ayudan a expulsar el exceso de sal. Pueden llegar a medir 20m, sin embargo, se conocen más como arbustos pequeños de $6 \mathrm{~m}$ de altura (Figura 5). Normalmente crecen en las orillas de las lagunas salobres o en desembocaduras de ríos con influencia de la marea. Además, se diferencia del mangle negro porque tiene una corteza con grietas (Mejía et al., 2014) 


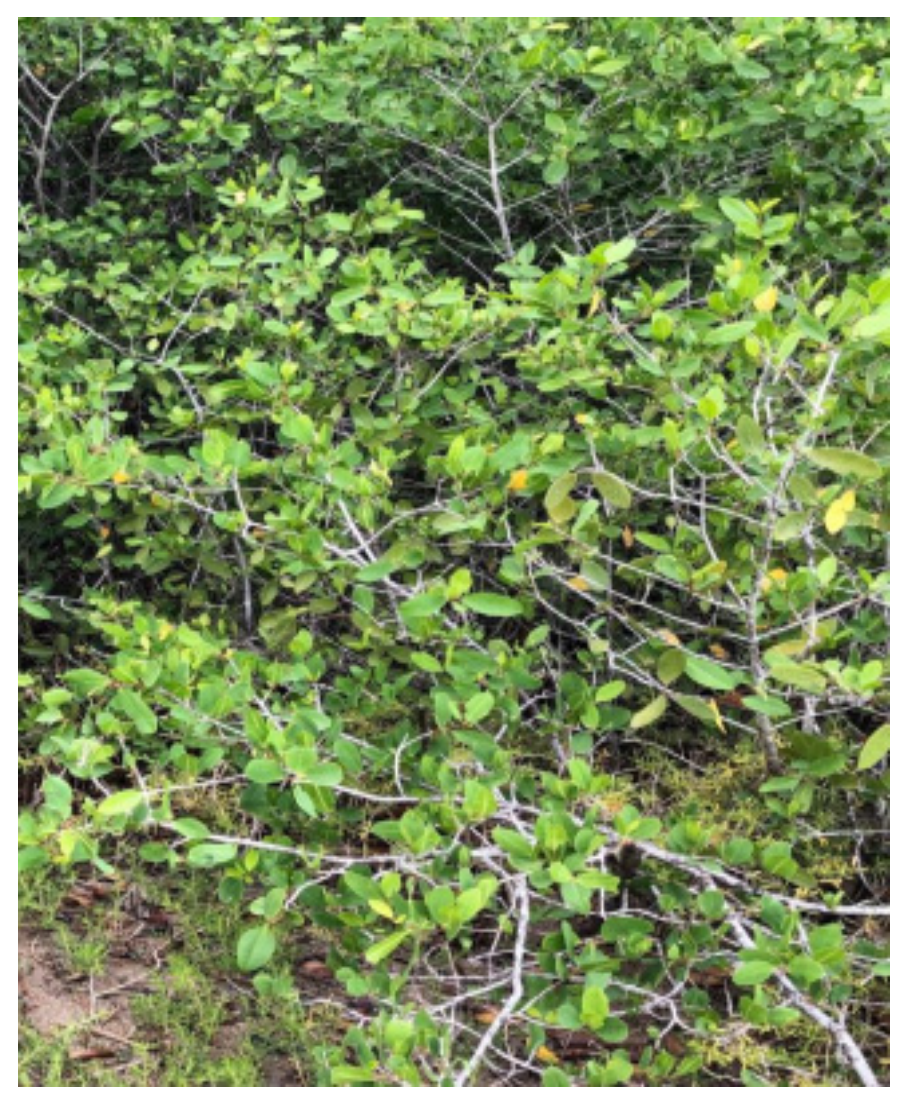

Figura 5. Mangle blanco

Fuente: Elaboración propia

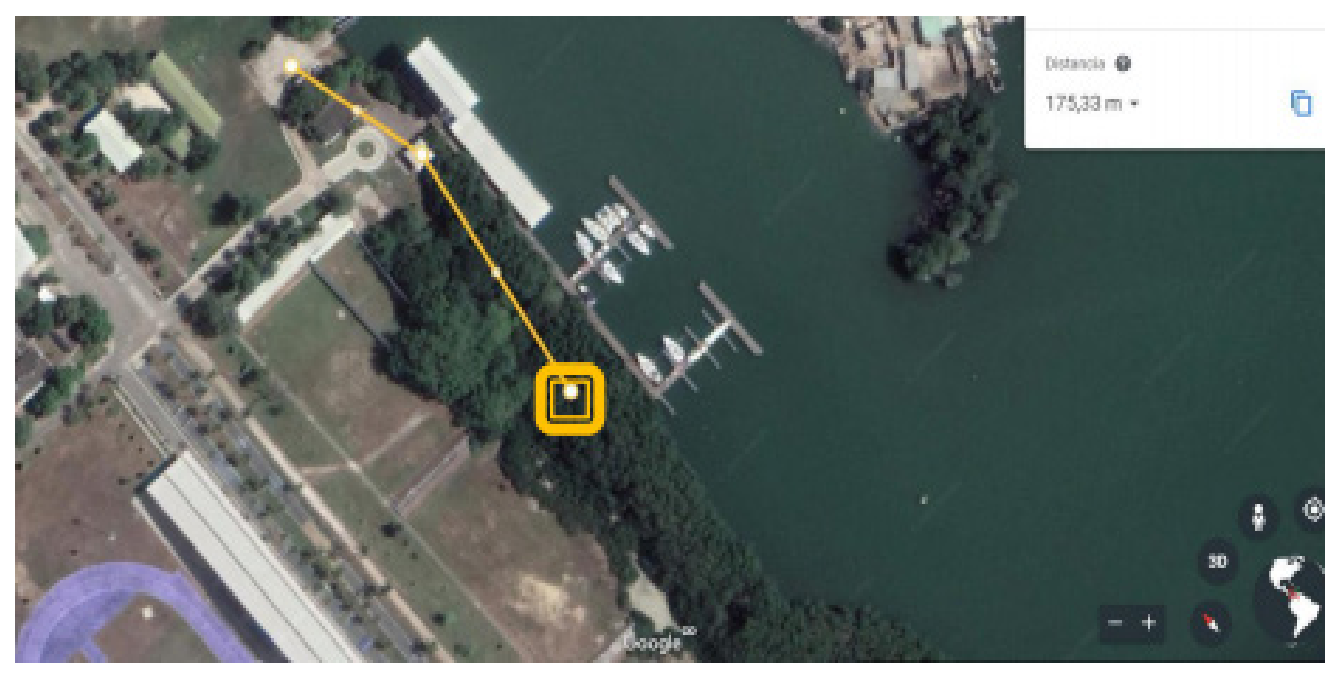

Figura 6. Tercera estación

Fuente: Elaboración propia con base en Google Earth (2019)

En cuarta estación se puede observar la transición de agua a tierra propia del ecosistema, gracias a la presencia de una pequeña laguna, en este sitio también se observaron aves como pericos (Eupsittula pertinax), gavilanes
(Rupornis magnirostris), garza blanca (Ardea alba), alcatraces (Pelecanus occidentalis). Cabe anotar que este sitio se encuentra ubicado en las coordenadas $10^{\circ} 23^{\prime} 08^{\prime \prime} \mathrm{N} 75^{\circ} 31^{\prime} 40^{\prime \prime} \mathrm{W}$ 
Diseño de un Sendero Ecológico como Estrategia Pedagógica para Promover el Conocimiento del Ecosistema de Manglar en la Escuela Naval de Cadetes "Almirante Padilla"

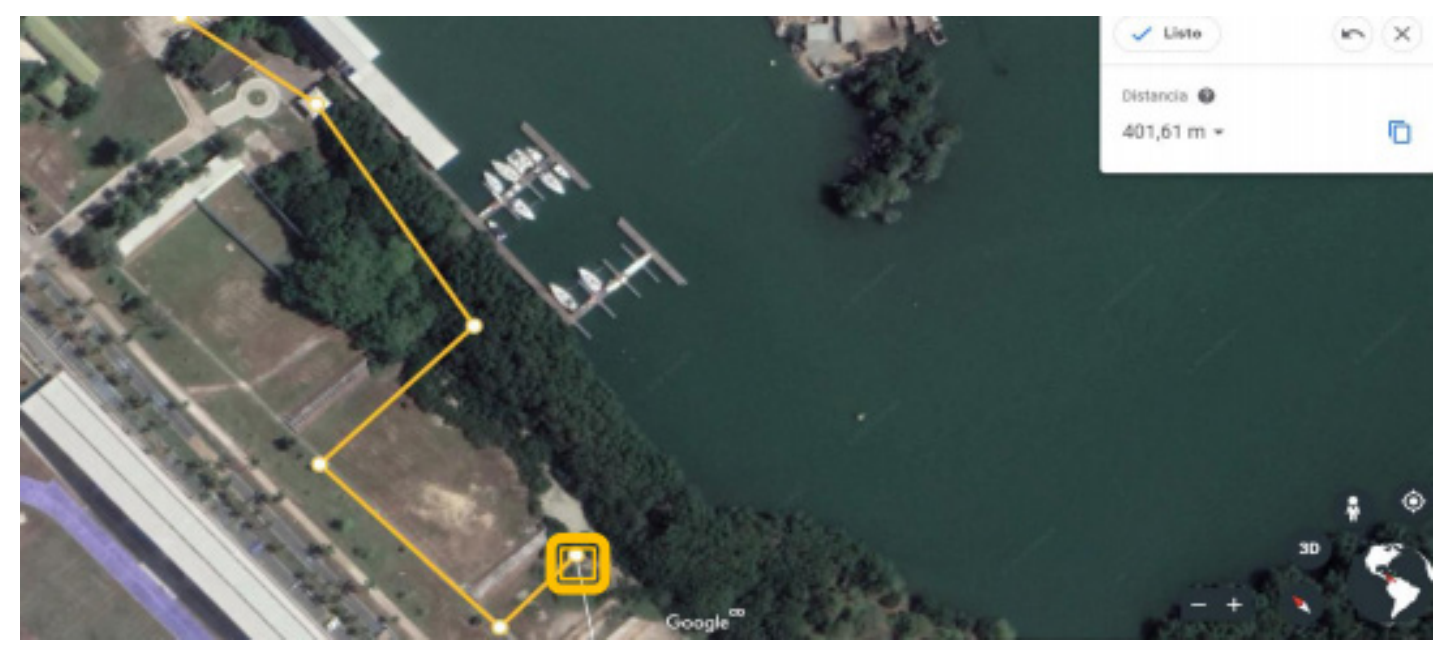

Figura 7. Cuarta estación

Fuente: Elaboración propia con base en Google Earth (2019)

En la quinta y última estación (Figura 9) se encuentra el mangle negro (Avicennia germinans) (coordenadas $10^{\circ} 23^{\prime} 06^{\prime \prime} \mathrm{N} 75^{\circ} 31^{\prime} 40^{\prime \prime}$ $\mathrm{W})$ : Se identifica porque tiene raíces poco profundas que crecen de la base del tronco en todas las direcciones con abundantes estructuras llamadas neumatóforos, que parecen pequeñas raíces que se levantan desde el suelo, y ayudan a captar el oxígeno del ambiente debido a que hay poco en el agua. Los árboles pueden alcanzar $20 \mathrm{~m}$ de altura y se caracterizan por tener una corteza interna amarrilla y una corteza externa enteriza y oscura. Sus frutos son ovalados y achatados en un extremo (Mejía et al., 2014) (Figura 8).

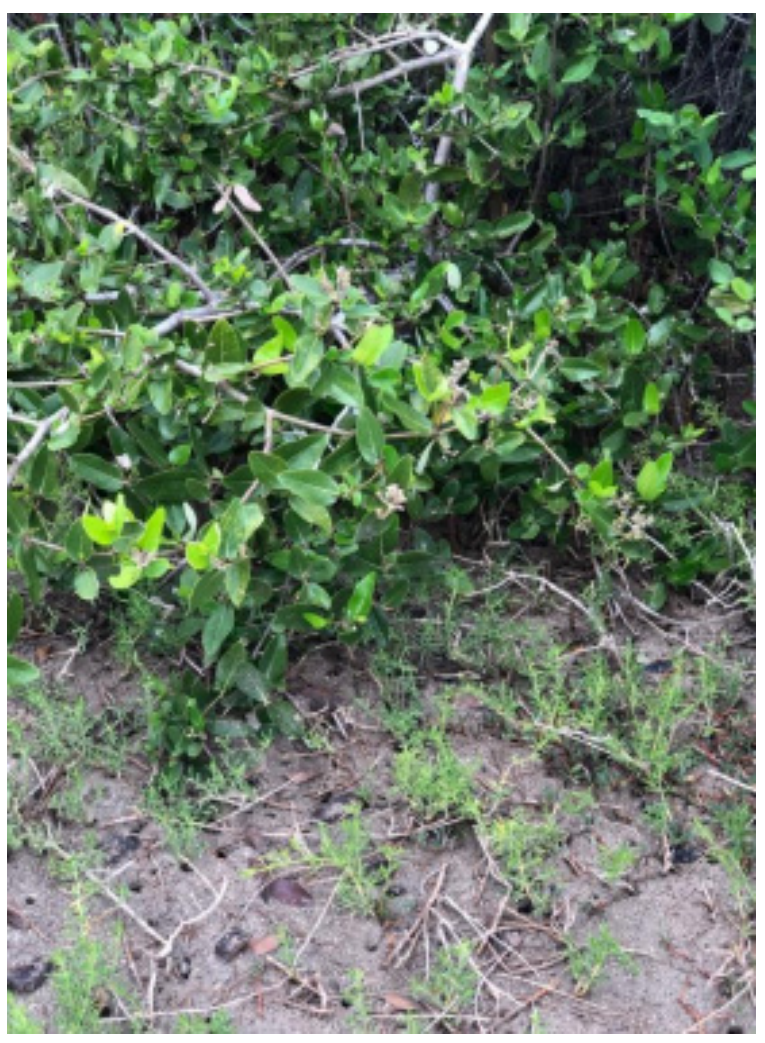

Figura 8. Mangle negro

Fuente: Elaboración propia 


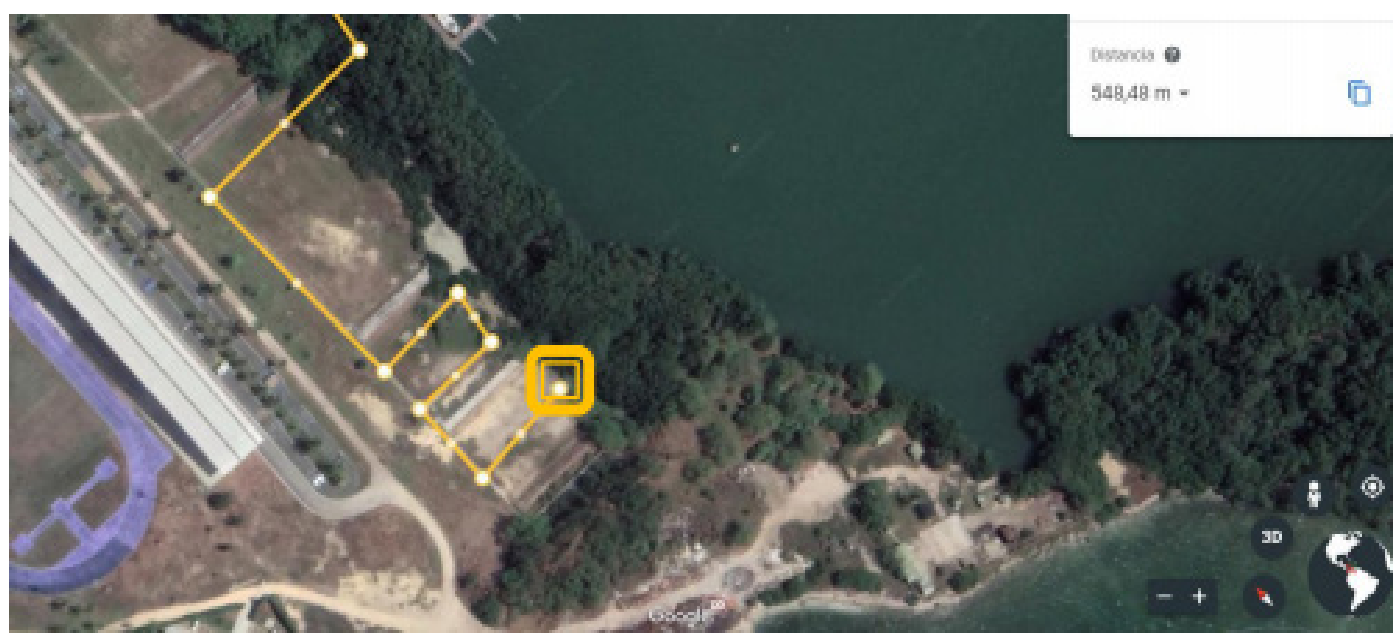

Figura 9. Quintas estación

Fuente: Elaboración propia con base en Google Earth (2019)

Cabe resaltar que el área recorrida y delimitada es de fácil acceso, no presenta zonas inundables, por lo que brinda la facilidad para el desplazamiento del personal, además de la observación en vivo de especies vegetales y animales propias del ecosistema, cumpliendo con lo que se requiere para la atención del per- sonal interesado en realizar el recorrido. Sin embargo, en algunos puntos se requiere retirar residuos orgánicos e inorgánicos de diferente naturaleza para facilitar aún más el acceso a las estaciones y evitar una mayor afectación al ecosistema (Figura 10).

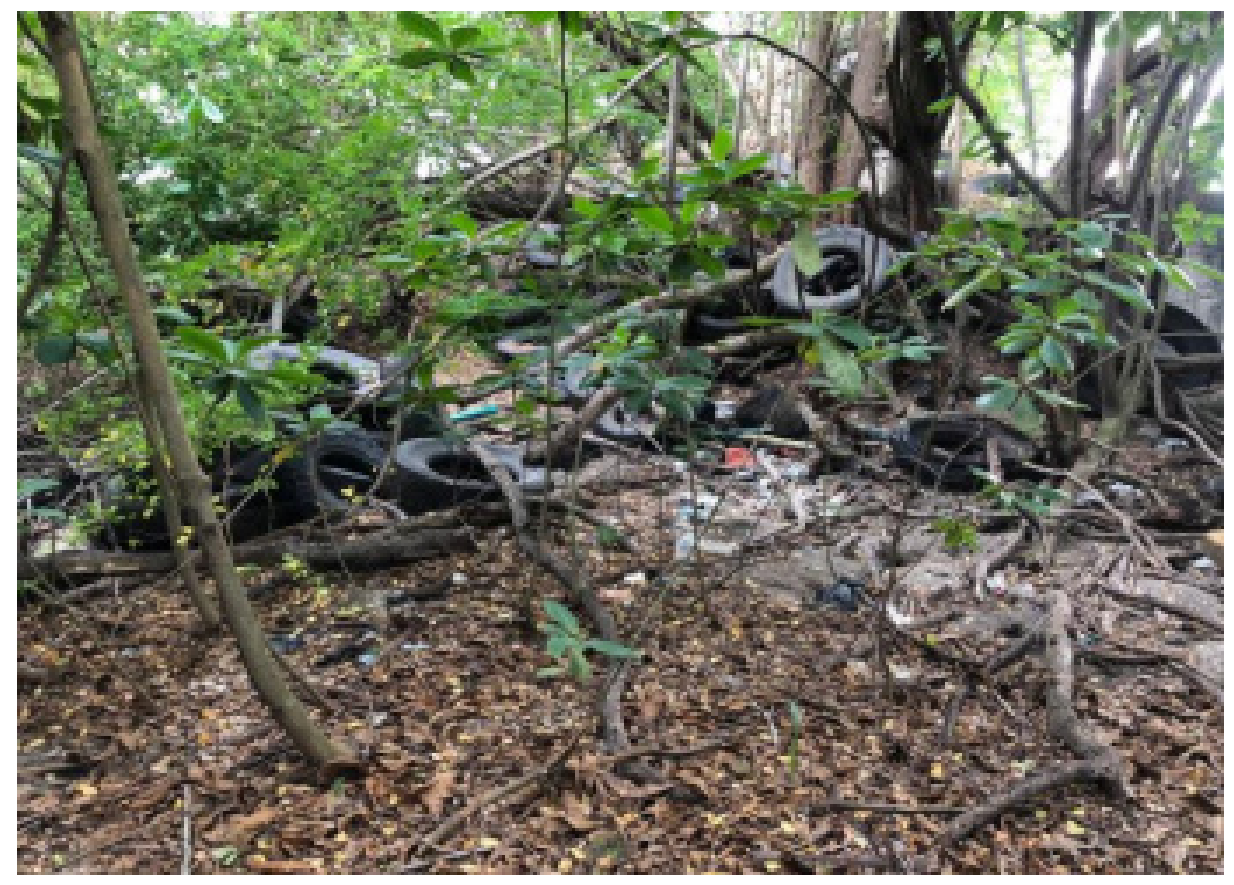

Figura 10. Desechos encontrados en la zona de estudio

Fuente: Elaboración propia

Finalmente, con la información levantada y las estaciones identificadas a continuación se presente el esquema del sendero en su totalidad ilustrando todo el recorrido en verde y distancia entre estaciones de color rojo (Figura 11). 
Diseño de un Sendero Ecológico como Estrategia Pedagógica para Promover el Conocimiento del Ecosistema de Manglar en la Escuela Naval de Cadetes "Almirante Padilla"

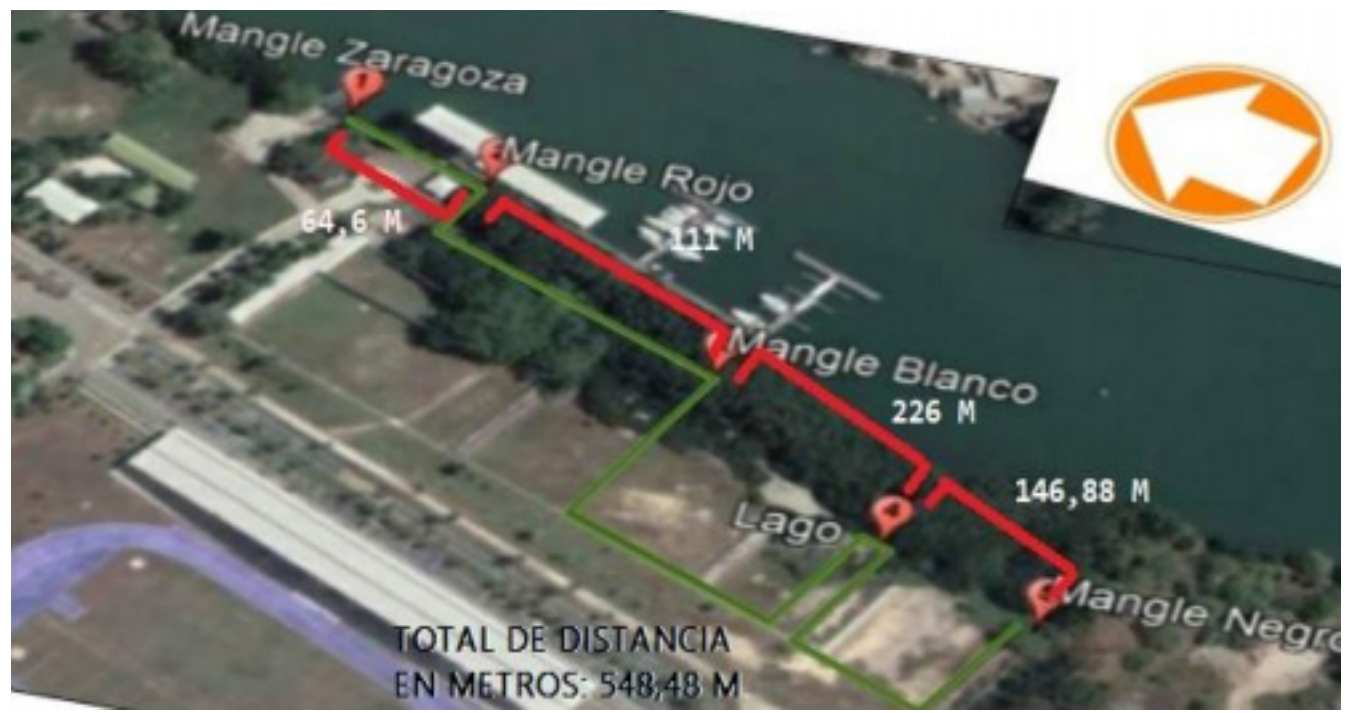

Figura 11. Sendero propuesto

Fuente: Elaboración propia

Como se observa en la figura 11, el diseño de sendero que se realizó tiene una longitud de 548.8 metros, y se caracteriza por ser de tipo inter-estación, es decir es el espacio que hay entre las estaciones interpretativas y que son recorridas por los visitantes. En las interestaciones también se pueden dar explicaciones por parte de un guía; así mismo es un sendero lineal o abierto ya que consta de un recorrido con inicio y final en diferente zona del área con modalidad de guiado ya que es conducido por un monitor y se sigue una ruta definida (Larbanois, 2004).

Por otra parte, se debe tener en cuenta que el sendero está ubicado en la parte Sur Este de la Isla Manzanillo, sin embargo es necesario saber que queda muy cercano al borde de costa en con un punto de inicio con orientación de Norte a Sur; el sendero se encuentra ubicado en proximidades al Pañol de botes, en donde se pueden ubicar amplios espacios para el manejo de personal, pudiendo manejar grupos de hasta 20 individuos.

Así mismo el recorrido consta de $45 \mathrm{~min}$ a 1 hora aproximadamente con el objetivo de que sea una actividad no tan extensa pero que posea la información necesaria y sea fructífera para las personas que la desarrollan; una vez se termine el recorrido en el punto 5 "Mangle Negro" el personal se dirige hacia el campo de paradas con el fin de recibir una charla de finalización y para resolver las dudas pertinentes. Así mismo, en ese punto y aprovechando la infraestructura de la zona, se podrá tener acceso a un punto de hidratación, el cual es importante para preservar la integridad del personal en términos de salud.

Es relevante anotar que los recorridos deberán ser encabezados por un guía asignado por la ENAP, quien será el encargado de brindar la mayor información posible del ecosistema de manglar, haciendo mucho más productiva la experiencia, así mismo se les capacitará en manejo de personal y manejo de contingencias (Arenguen y De Añez, 1995), para ello se debe contar con una fase de preparación de Cadetes o Guardiamarinas interesados, quienes recibirán instrucciones precisas para el desarrollo de la actividad por parte de los profesionales de la biología con los que cuenta la escuela. Siendo por lo tanto un sendero con recorrido guiado.

\section{Conclusiones}

Teniendo en cuenta la información recopilada durante las dos salidas de campo se evidencia gran presencia de residuos inorgánicos y orgánicos, que llegan no solo del sector del Zapatero, que es el más cercano a la Isla de Manzanillo sino que se evidencian desechos propios de las actividades de la ENAP. Con base en esto es necesario realizar una limpieza que permita la favorabilidad del desarrollo del sendero, y la reproducción de los manglares. 
Después del desarrollo del recorrido, se pretenden implementar 5 estaciones en las cuales se abordan el reconocimiento y el análisis de distintas especies de manglar presentes en la Isla de Manzanillo y la fauna característica del ecosistema, con el objetivo de que las personas que vayan a utilizar el obtengan una información integral de la dinámica del ecosistema, generando transmisión de conocimiento y conciencia de conservación.
El sendero que se estructuró fue un sendero de forma lineal ya que cuenta con un punto de inicio y uno de terminación sin que haya alguna conexión entre los mismos, se eligió ya que es lo más favorable para realizar el recorrido teniendo en cuenta las condiciones del terreno, distribución del manglar y los puntos de las estaciones.

\section{Referencias}

Autocad. (1982). AUTOCAD ya disponible con herramientas especializadas. http://www.3dcadportal.com/autocad.html

Ávila, H. (2006). Introducción a la metodología de la investigación. https://clea.edu.mx/biblioteca/INTRODUCCION\%20A\%20LA\%20METODOLOGIA\%20DE\%20LA\%20INVESTIGACION.pdf

Fernández, S. (27 de mayo de 2002). Investigación cuantitativa y cualitativa. https://www.fisterra.com/ gestor/upload/guias/cuanti_cuali2.pdf

Fuentes. (2015). Cuaderno de campo. https://www.researchgate.net/publication/278678399_Cuaderno_de_campo

Fundación Universitaria Tecnológico Comfenalco. (2010). Mangles de Cartagena de Indias. http://observatorio.epacartagena.gov.co/wpcontent/uploads/2016/10/MANGLES-DE-CARTAGENA.pdf

Google Earth (2019). Google Earth. https:/www.google.com/intl/es/earth/

Larbanois, G. d. (2004). Guía para el diseño y operación de senderos interpretativos. https://www.entornoturistico.com/wpcontent/uploads/2017/04/Gu\%C3\%ADa-para-el-dise\%C3\%B1o-y-operaci\%C3\%B3nde-senderos-interpretativos.pdf

Lechner, L. (2004). Planificación, construccion y mantenimiento de senderos en áreas protegidas. https://ppduruguay.undp.org.uy/images/OtrasPublicaciones/UsoPublAreasProtegidas/Trail_manual_es\%20Lechner.pdf

Mejía L., Molina M., Sanjuán A., Grijalba., M., Niño L. (2014). Bosque de manglar: Un ecosistema que todos debemos cuidar. http://observatorioirsb.org/cmsAdmin/uploads/cartilla-manglar-28pg-(1)_001.pdf

Mendoza y Vitola. (octubre de 2014). ARMAS BIOLÓGICAS RIESGO LATENTE.UNA REVISIÓN SISTEMÁTICA DE LA LITERATURA. Derrotero Escuela Naval. http://revistas.escuelanaval.edu.co/index.php/DERROTERO/article/view/78/55

Ministerio de Medio Ambiente. (2002). Uso sostenible, manejo y conservación de los ecosistemas de manglar en Colombia. http://www.minambiente.gov.co/index.php/component/content/article/117-manejosostenible-de-ecosistemas-marinos-y-costeros-y-sus-servicios

Quintana y Montgomery. (2006). Metodología de Investigación. Metodología de Investigación. https://cienciassociales.webcindario.com/PDF/Cualitativa/Inv_quintana.pdf

Riosmena, R. (15 de julio de 2015). El calentamiento global está afectando a los manglares. https:// www.bcsnoticias.mx/el-calentamiento-global-esta-afectado-a-los-manglares-debaja-california-sur/ 